Cahiers $d u$ MONDE RUSSE

\section{Cahiers du monde russe}

Russie - Empire russe - Union soviétique et États indépendants

$57 / 4 \mid 2016$

Varia

\title{
Bakst penseur et écrivain : en russe et en français
}

\section{Olga Medvedkova}

\section{OpenEdition \\ Journals}

Édition électronique

URL : http://journals.openedition.org/monderusse/10042

DOI : $10.4000 /$ monderusse. 10042

ISSN : 1777-5388

Éditeur

Éditions de l'EHESS

\section{Édition imprimée}

Date de publication : 1 octobre 2016

Pagination : 975-979

ISBN : 978-2-7132-2542-0

ISSN : $1252-6576$

\section{Référence électronique}

Olga Medvedkova, «Bakst penseur et écrivain : en russe et en français », Cahiers du monde russe [En ligne], 57/4 | 2016, mis en ligne le 01 octobre 2016, Consulté le 25 septembre 2020. URL : http:// journals.openedition.org/monderusse/10042 ; DOI : https://doi.org/10.4000/monderusse.10042 
the book, some are noticeably absent such Soviet food ways, fashion, leisure, and religious practices. I mention this, of course, not as a criticism, but to emphasize Sheila Fitzpatrick's point that "historians have often privileged other aspects of life, but it is the everyday we all live in. Elusive though it may be, we cannot afford to leave it outside our field of vision" (p. 402). Point well taken.

Donald J. Raleigh

University of North Carolina, Chapel Hill

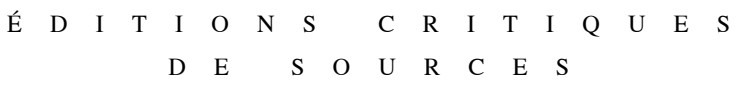

Bakst penseur et écrivain : en russe et en français

Elena TERKEL', Džon E. BOULT [John E/ Bowlt], O. KOVALEVA, éds.

\section{Lev Bakst, Moja duša otkryta}

[Mon âme est ouverte)],

Vol. 1 : Stat'i ; Žeskaja pervaja ljubov'(roman) ; Libretto [Articles ; Ce cruel premier amour (roman); Libretto], 350 p.; vol. 2 : Pis'ma [Correspondance], 400 p. Moscou : Iskusstvo 21 vek, 2012, 2 vols.

Jean-Louis BARSACQ, traduction et présentation de

Léon Bakst

Correspondance et morceaux choisis

Paris : L'Âge d'homme, 2016.

En 2012 paraissait à Moscou le livre intitulé Moja duša otkryta [Mon âme est ouverte] ; il contenait les écrits de Léon Bakst (1866-1924). Les deux volumes, de 350 et 400 pages, changeaient notre compréhension de l'artiste. Ce dessinateur habituellement qualifié d'exquis et de sensuel, ce décorateur au don pour les couleurs reconnu qui avait, certes, révolutionné la scène russe et occidentale de la première moitié $\mathrm{du} \mathrm{Xx}^{\mathrm{e}}$ siècle, cet artiste quelque peu énigmatique, aux penchants tout autant néoclassiques qu'exotiques, se révélait un homme de lettres et un intellectuel accompli, articulant comme peu d'artistes autour de lui, c'est-à-dire avec force et clarté, quantité de problèmes artistiques, psychologiques et politiques de son époque.

Les historiens de l'art avaient déjà une idée de cet héritage littéraire et théorique laissé par Bakst, grâce notamment à ses articles publiés dans la presse russe et française. Ils connaissaient le livre d'Irina Pružan $(1975)^{1}$, fondé sur les sources et contenant nombre d'importantes citations des écrits de l'artiste, ainsi que les publications d'Il'ja Zil'berštein dans la collection « Héritage littéraire », parues entre 1968 et 1982. C'est qu'en 1965, le fils de Bakst et de Lubov' Gricenko, née Tret' jakova (1870-1928), André Bakst (1907-1972), avait donné les archives de 
son père à la Galerie Tretiakov, où ces quelques 2620 pièces $^{2}$ sont désormais conservées au département des manuscrits (fonds 111 ) $^{3}$.

Néanmoins l'ouvrage paru en 2012, précédé d'un article de John Bowlt et Elena Terkel $^{4}$, a été une véritable découverte. Tout d'abord, les lecteurs - et pas seulement les historiens de l'art - peuvent désormais savourer le roman inédit de Bakst Ce cruel premier amour et apprécier son style personnel, original, précis et franc, ennemi des clichés. Ce même premier volume contient plusieurs articles ainsi que les scénarios et les livrets inédits de Bakst. Ensuite, un volume entier, le second, contient 230 pages de sa correspondance, aussi bien intime que littéraire et philosophique, soigneusement commentée par Elena Terkel' et Anna Černuhina ${ }^{5}$.

Seul manque étonnamment de cet ensemble, l'essai de Bakst Serov et moi en Grèce, publié en émigration, aux éditions berlinoises Slovo, en 1923, en russe, un an avant la mort de l'artiste. Cet essai, parmi les plus beaux morceaux de la prose russe symboliste, apprécié par Ivan Bunin et Zinajda Gippius, réédité en 1971 en russe dans un recueil consacré à Valentin Serov ${ }^{6}$, vient de paraître, quasi en même temps, en deux traductions indépendantes, italienne et française ${ }^{7}$. Dans le contexte des autres écrits de Bakst et de ses lettres - notamment celles adressées à Nouvel $\left(\text { Nuvel }^{\prime}\right)^{8}$, Annenskij et Rozanov -, ce texte donne une véritable clé d'accès au tableau de Bakst « Terror antiquus » et plus largement à la synthèse stylistique de l'Antiquité et de l'Orient que l'artiste opère, dès 1909, dans les premiers spectacles des Ballets russes. Mais même sans ce texte, l'édition des écrits de Bakst, parue en 2012, est un événement ; l'ouvrage est devenu depuis incontournable pour tout spécialiste de la culture russe de la fin du XIX ${ }^{\mathrm{e}}$ et du début du XX $\mathrm{X}^{\mathrm{e}}$ siècle. De ce point de vue, l'historien de l'art occidental non-russophone, et en particulier français, était jusqu'à présent beaucoup moins bien servi.

Pour remédier à cette lacune, le livre intitulé Léon Bakst, Correspondance et morceaux choisis, paraît, enfin, chez l'Âge d'homme en 2016 ; il est réalisé, comme l'annonce dans le préambule Jean-Louis Barsacq, essentiellement à partir de l'édition russe de 2012. Dans ce même préambule, il qualifie son travail de « traduction-adaptation ». Nous ne saurons rien d'autre sur la façon dont le traducteur adapte les écrits de Bakst. De fait, après un bref « Résumé de la vie de Léon Bakst » (p. 15-18) et un autre texte aussi bref, intitulé «L'envers du décor » (p. 19-20), qui nous apprend que « l'artiste Léon Bakst, adulé et acclamé, eut une carrière triomphale, mais sa vie contrastée comporta de sévères dépressions nerveuses ${ }^{9}$ », le lecteur francophone consulte un choix de lettres de Bakst, séparées en cinq chapitres, puis huit « morceaux choisis » de ses « essais-chroniques-romans $»^{10}$, tout en ignorant pourquoi ces lettres-là ou ces textes-là sont choisis et pas les autres. Ou, question plus importante, pourquoi tel morceau est traduit et tel autre coupé, car chaque lettre sélectionnée contient de nombreuses coupures, certes indiquées par des crochets, mais nullement expliquées : ces coupures enlèvent parfois jusqu'à un tiers du texte. 
Mais la plus extraordinaire surprise attend celui qui lirait cette traduction en regard avec le texte russe. Prenons, au hasard, une lettre, celle écrite par Bakst de Paris le 20 juillet 1893 à son ami Alexandre Benois. Voilà ce que Bakst écrit en russe :

Французские художники думают и сильно тоскуют, но они впадают
в крайнюю ошибку: они слишком веротерпимы стали (если можно
так выразиться). Всякое направление, всякий манер, всякий трюк и
оригинальничанье - все имеет свой raison d'être, и они с каждым
художником в этом роде страшно нянькаются, точно с лихорадочным,
капризным ребенком, заботливо объясняя всякую чепуху, выходку, мазок
- болезненно-художественным состоянием души... Они перехватили...

En voici la traduction :

Le milieu artistique en France, accorde une place excessive aux nouvelles tendances, aux infléchissements, aux trucs et aux originalités de toutes sortes. Pour les artistes ces tendances auraient chacune sa raison d'être, il faudrait donc les protéger et les encourager. On se comporte ici comme le feraient des nourrices chargées d'enfants fiévreux et capricieux, et on explique soigneusement chaque bêtise, chaque inflexion, chaque gribouillage $[\ldots]^{12}$.

Le lecteur bienveillant passera sur ces nourrices qui surgissent, absentes chez Bakst, ainsi que sur l'imprécision générale de la traduction. Mais pourquoi amputer l'argument final de Bakst : « en expliquant chaque absurdité, plaisanterie, touche de pinceau par un état d'âme artistique douloureux... »? Pourquoi priver Bakst de sa force, de sa capacité non seulement d'observer, mais aussi de comprendre et d'expliquer les phénomènes ? L'artiste y perd, hélas, en intelligence.

Dans d'autres cas, les coupures et imprécisions semblent encore plus incompréhensibles. Par exemple, la lettre de Bakst à Walter (Valentin) Nouvel datant du 15 octobre 1908 n'est pas traduite, seulement celle du 20 octobre. Or, le propos que Bakst développe dans cette dernière est le prolongement de la réflexion lancée dans la lettre précédente et consiste en une synthèse sophistiquée entre la culture juive et la culture grecque. L'adaptateur l'a sacrifié : pour quelle raison ? Le propos de Bakst, réduit de moitié, devient vague, à peine intelligible. Quant à la traduction de la lettre du 20 octobre, elle est par moment tout simplement fautive. La lettre originale commence ainsi :

Ты все-таки неправ и, оправдываясь косвенно, нападал на меня. Чем провинилось еврейское миросозерцание?

Ceci est traduit en français de la manière suivante :

Tu as tout de même tort ! En te justifiant indirectement, tu reportes la responsabilité sur moi. À qui la faute si les Juifs ont une bougeotte $?^{13}$

Mirosozercanie, la « vision du monde »- mot-calque de l'allemand Weltanschauung, très courant dans le vocabulaire des Russes cultivés depuis la fin du $\mathrm{XIX}^{\mathrm{e}}$ siècle - est traduit par «bougeotte ». 
Dans la même lettre, le mot « элленизм [hellénisme] » est traduit par « la religion des Grecs anciens ». L'hellénisme chez Bakst est comparé avec le judaïsme (иудаизм). Utiliser ces deux notions, l'une culturelle et l'autre religieuse, comme deux éléments comparables, à l'intérieur d'une formule - « Но огромная пропасть элленизма и иудаизма - еще вопрос [Mais le hiatus énorme entre l'hellénisme et le judaïsme reste discutable] » - signifie que l'un de ces éléments change de couleur. Soit l'hellénisme est considéré par Bakst comme un élément religieux, soit le judaïsme comme un élément culturel, soit, enfin, tous les deux prennent une connotation intermédiaire, entre le religieux et le culturel et deviennent « vision du monde (mirosozercanie) ». Un long passage où Bakst parle ensuite du monothéisme des Grecs est tout simplement coupé par le traducteur.

Nous nous arrêterons là, car on l'aura compris. Si nous devons nous réjouir de toute tentative de rendre Bakst, artiste cosmopolite qui a longtemps vécu et travaillé à Paris, au lecteur francophone, nous devons au même lecteur, et tout spécialement au lecteur non russophone, un avertissement que voici : le livre de sa correspondance et de ses écrits choisis, récemment traduits en français, ne peut en aucun cas être utilisé en tant que source académique.

1 - Irina Pružan, Lev Samojlovič Bakst, M. : Iskusstvo, 1975.

2 - 140 autres pièces se trouvent dans les archives du Musée russe à Saint-Pétersbourg (A.A. Prokofi'ev, « Iz perepiski L. Baksta s A. Benua, 1891-1897 (po materialam otdela rukopisej GRM) [Extraits de la correspondance de L. Bakst avec A. Benois (d'après les fonds du cabinet des manuscrits du Musée russe], Peterburgskij rukopisnyj sbornik [Recueil pétersbourgeois des manuscrits], fasc. 4, 2001, p. 357-366, ainsi que dans d'autres archives russes et occidentales.

3 - Sur les archives littéraires de Bakst, voir : John E. Bowlt, Anna Černuhina, Olga Kovaleva, Elena Terkel", " "Worlds of Magic": The Literary Heritage of Léon Bakst », The Tretyakov Gallery, 1, 2008, p. 78-81 (en russe et en anglais). Dans le même numéro : un premier choix des lettres de l'artiste. À propos des différents lieux de conservation des archives de Bakst, voir également l'introduction « Au lecteur », in Lev Bakst, Moja duša otkryta, t. 1, p. 5-12.

4 - « Literaturnoe tvorčestvo Baksta [L'Euvre littéraire de Bakst] », in Lev Bakst, Moja duša otkryta, t. 1, p. 13-49.

5 - Lev Bakst, Moja duša otkryta, p. 243-322 : commentaires.

6 - I. Zil’berštejn, V. Samkov, dir., Valentin Serov v vospominanijah, dnevnikah i perepiske sovremennikov [Valentin Serov à travers les Mémoires, les journaux intimes et la correspondance de ses contemporains], L., 1971, p. 562-587.

7 - Valentina Parisi, éd. et trad., Lev Bakst, In Grecia con Serov : Appunti di viaggio (In Greece with Serov. Travel Notes), Milan : Excelsior 1881, 2012 ; Léon Bakst : Serov et moi en Grèce (1923), traduit du russe et introduit par Olga Medvedkova, préface de Véronique Schiltz, P. : Triartis, 2014.

8 - Voir surtout deux lettres des 15 et 20 octobre 1908, Lev Bakst, Moja duša otkryta, t. 2, p. 141-146.

9 - Ibid, p. 20.

10 - À quoi dans les annexes sont ajoutées trois lettres de Cocteau à Bakst, p. 397-399, sans indication de leur provenance, un « Historique des spectacles auxquels Léon Bakst a contribué (1902-1912) », ainsi que l'index dans lequel des surprises nous attendent : nous y trouvons par exemple un(e) certain(e) Ossipovna, Rebikov, alors que dans la lettre originale il s'agit de deux personnes, M. Rebikov et Luiza Osipovna. 
11 - Lev Bakst, Moja duša otkryta, t. 2, p. 12.

12 - Léon Bakst, Correspondance et morceaux choisis, traduction et présentation de Jean-Louis Barsacq, P. : L'Âge d'homme, 2016, p. 32.

13 - Ibid., p. 167.

\section{Olga Medvedkova \\ CNRS-ENS}

\section{Arkadi ZELTSER, éd.}

\section{To pour out my bitter soul Letters of Jews from the USSR 1941-1945}

Jérusalem : Yad Vashem, 2016, bibliographie, index, 276 p.

En dépit des travaux publiés récemment sur les massacres de masse en URSS durant la Seconde Guerre mondiale ${ }^{1}$, en dépit également des recueils passionnants sur ce sujet édités régulièrement par l'organisation russe « Centr i Fond "Holokost" »" de nombreux aspects de la vie quotidienne de la population et des territoires occupés pendant le conflit, et tout particulièrement des Juifs de Russie, restent à ce jour peu ou mal connus. Cette lacune est partiellement comblée par le présent ouvrage édité par le Mémorial de Yad Vashem. Les cent lettres qu'il contient sont réparties thématiquement dans sept chapitres intitulés « Dernières lettres », «Premières réactions aux meurtres de masse », « Réactions à l'antisémitisme », «Vie juive à l'arrière », «Vie juive dans les territoires libérés », «Le patriotisme soviétique et l'image du soldat juif », «Le destin des unités militaires juives dans l'Armée rouge ». À l'intérieur de chaque partie, les lettres, traduites en anglais du russe et du yiddish et dont il aurait été passionnant de disposer des textes originaux, sont classées chronologiquement. L'ensemble est précédé d'une introduction précise et stimulante rédigée par A. Zeltser. Ces cent lettres proviennent du fonds Yad Vashem et du fonds du Comité juif antifasciste (aux Archives d'État de la Fédération de Russie, GARF), auxquels un nombre important de destinateurs juifs s'adressa tout au long de la guerre. Elles s'inscrivent dans la même approche « juive » de la guerre que celle qui avait prévalu il y a de cela quelques années pour la publication d'une série de livres consacrés à la correspondance entre des Juifs soviétiques et l'écrivain Il'ja Erenburg $^{3}$ et des lettres envoyées par des soldats soviétiques juifs à leurs familles depuis le front. Cependant, par rapport à ces publications, To pour out my bitter soul, qui comporte un nombre substantiel de lettres envoyées à Erenburg, apporte une nuance essentielle sur la judéité affirmée des auteurs, judéité qui s'exprime dans la « chair » même des lettres, que ce soit par des références aux fêtes juives et à la religion, l'utilisation imparfaite du russe ou l'emploi du yiddish. Outre les moyens linguistiques, comme le choix d'une langue juive, l'affirmation d'une fierté juive prend parfois des formes touchantes et naïves. C'est le cas dans un certain nombre de lettres dont les auteurs énumèrent les figures historiques du judaïsme universel. Ainsi, dans la lettre 87, le destinateur loue la grandeur du « peuple juif 